\title{
Post-BPPV Syndrome: Our Experience in a Specialized Neurotology Clinic in Kolkata
}

\author{
Anirban Biswas ${ }^{1} \quad$ Nilotpal Dutta ${ }^{1}$ \\ ${ }^{1}$ Vertigo and Deafness Clinic, Kolkata, India
}

Address for correspondence Anirban Biswas, MBBS, DLO, Consultant Neurotologist, Vertigo and Deafness Clinic, BJ 252 Salt Lake, Kolkata 700091, India (e-mail: vertigodeafnesstinnitus@gmail.com).

\begin{abstract}
Keywords

- post BPPV

- residual dizziness

- light headedness

- unsteadiness

- Canalith repositioning Manoeuvre

Introduction The common cause of approximately 25 to $30 \%$ of all patients presenting to the neurotologist with the complaint of head-spinning is benign paroxysmal positional vertigo (BPPV) that is the commonest cause for which a patient attends a vertigo clinic. Though BPPV is very effectively treated with the canalith repositioning maneuver (CRM), a considerable percent of these patients (34-61\% as per different studies $)^{7-10}$ is not completely symptom-free even after a very successful maneuver (s) and complain of a new set of symptoms of light-headedness, unsteadiness, or dizziness or a combination of them termed as post-BPPV syndrome or as residual dizziness (RD) after successful correction of BPPV. Post-BPPV syndrome induces a very poor quality of life and is very incapacitating to most patients who suffer from it.

Materials and Methods In Vertigo and Deafness Clinic, Kolkata, a total number of 200 patients were diagnosed with geotropic variety of BPPV in a period of 1 year from April 1, 2018 to March 31, 2019. Out of these 200 patients, the study was conducted on 178 patients who came for follow-up and also underwent all the necessary vestibular function tests. The remaining 22 patients who did not turn up for follow-up or did not undergo the tests either due to financial constraints or lack of time were excluded from the study group. The study was limited only to patients who had geotropic nystagmus and in patients with ageotropic nystagmus who were excluded from the study. One hundred seventy-eight patients of BPPV who were included in the study were managed by a protocol elaborated below devised by the first author.

Results Following this protocol of BPPV management, only 23 (13\%) patients after successful CRM presented with post-BPPV syndrome that is much less than the international figures of 34 to $61 \%$. Analysis of data also showed that there was a huge psychic component in post-BPPV syndrome and that there was practically no organic vestibular deficit in post-BPPV syndrome.

Conclusion Following our protocol of management of BPPV patients, which does not involve any new maneuver, only $13 \%$ of the patients complained of RD after the successful repositioning maneuvers. This is far lesser than the published international figures and this protocol may hence be tried and followed by other neurotology centers too. This protocol drastically reduces the morbidity of patients after the BPPV has been
\end{abstract}

DOI https://doi.org/ 10.1055/s-0039-1695666 ISSN 2581-9607
Copyright @2019 Indian Society of Otology
License terms

() (1) $\ominus \circledast$ 
corrected by the requisite maneuvers. In our group of 23 patients who had post-BPPV syndrome out of 178 patients who had BPPV, the symptoms subsided spontaneously without medication within 3 weeks in more than $70 \%$ of patients. Only in three (13\%) patients of post-BPPV syndrome, the RD persisted up to 12 weeks where drugs (selective serotonin reuptake inhibitor, benzodiazepines) and professional psychological counseling were required. None of these patients required any vestibular sedatives or the so called antivertigo drugs for amelioration of symptoms. It may hence be concluded that management of BPPV by this protocol reduces the incidence of post-BPPV syndrome and that antivertigo drugs have no role in the management of post-BPPV syndrome.

\section{Introduction}

The common cause of a patient to visit a vertigo clinic is head spinning due to benign paroxysmal positional vertigo (BPPV) that is presented by approximately 25 to $30 \%$ of all patients. ${ }^{1,2}$ It has a lifetime prevalence of $2.4 \%$ in the general population. ${ }^{3}$ BPPV is caused by the displacement of otoconia from the otolith organs, mainly the utricle into one or more of the semicircular canals. It is characterized by episodes of head spinning for few seconds only on change of head position (lying down in bed, getting up from bed. bending down, turning head up, turning from one side to other) sometimes even with nausea and vomiting. ${ }^{3}$ Possible etiological factors that have been proposed are head trauma, vestibular neuritis, and vascular disorders ${ }^{4-6}$ In most, if not in all patients, BPPV is very effectively corrected by a special form of physical therapy called the canalith repositioning maneuver (CRM). CRM consists of repositioning the otoconia that has been dislodged from the utricle and has entered the semicircular canal(s) back into the utricle. However, a considerable percent of patients $(34-61 \%)^{7-10}$ as per different studies is not completely symptom-free even after a very successful maneuver (s). They are of course relieved of the symptom of sudden severe head spinning on change of head posture after the properly done maneuver; however, they come back to the doctor with a new set of symptoms, namely unsteadiness, light headedness, and feeling of persistent dizziness, often a mix of all three even though there is no evidence of any positional vertigo whatsoever. Repeated positional tests show that the BPPV has been completely corrected, and hence there is no question of incomplete correction of the BPPV by the repositioning/ liberatory maneuvers in these patients. But the new set of symptoms is very distressing to most such patients and the morbidity as well as poor quality of life (QOL) caused by these symptoms is formidable if not intimidating to the patient. Their main complaint is that though change of head position is no longer causing any head spinning yet they have not yet got back their original stability and are in great discomfort as regards their balance. Several such patients cannot even convey their problem properly and just say that their balance is not normal. They feel they may lose balance and may fall but have never fallen. Morbidity from this symptom is very high and most patients have a very poor and miserable QOL. This is termed as post-BPPV syndrome. Most of these patients are put on long-continued vestibular sedatives and antivertigo drugs that do more harm than good to the patients.

\section{Aims and Objectives}

The aim of this study is to establish that by following a structured way of management as per the protocol (based on the possible pathomechanisms) followed in our clinic, the incidence of this neurotological disorder with high morbidity can be drastically reduced. In addition to this, the purpose of this article is to discuss the incidence, manifestations, presumed pathophysiology, predisposing factors and suggest a rational management of residual dizziness (RD) after BPPV. The objective is also to create awareness on this considerably common but under-recognized disease called the post BPPV syndrome or RD after successful CRM.

\section{Materials and Methods}

A cross-sectional study was done on 178 patients diagnosed with BPPV from April 2018 to March 2019 at Vertigo and Deafness Clinic, Institute of Neurotology, Kolkata, India. The diagnosis of BPPV was made first from the history of head spinning for a few seconds only on change of head position (i.e., during lying down or getting up from bed, turning from one side to another in supine position, bending downwards, looking upwards) and then on the basis of the diagnostic tests such as Dix Hallpike (for posterior canal BPPV), supine roll test (for lateral canal BPPV), and the deep head hanging test (for anterior canal BPPV). Dix Hallpike, supine roll, and deep head hanging tests were considered positive if nystagmus appeared with a latency at appropriate head positions during the diagnostic tests. Only patients with geotropic nystagmus were included in the study and patients who had ageotropic/apogeotropic nystagmus were excluded as there is some controversial opinion on the genesis of ageotropic nystagmus. Patients having other known causes of vertigo at the time of the clinical tests too were excluded from the 
study. There were some patients included in the study who had past histories very typical of Meniere's disease or that of vertiginous migraine but during the time of examination the patients were not having any active manifestations of these diseases. The etiological factors leading to BPPV were identified as much as possible and were divided into idiopathic, due to aging,, ${ }^{11,12}$ head trauma (if symptoms appeared within 10 days of any head injury), ${ }^{13}$ ototoxic drugs, migrane, ${ }^{7}$ and vestibular neuritis ${ }^{13}$. Once diagnosed, the requisite liberatory maneuver to reposition the canaloliths back into the otolith system was done in the same session. The initial symptoms of rotatory vertigo for few seconds only on the change of head position with nystagmus were then again revaluated after 72 to 96 hours (i.e., on the 3rd to 4th day depending on the patient's convenience) for any persistence of the features of positional vertigo, which if present were again treated with a repetition of the CRM till the symptoms of this rotatory vertigo on assuming the provocating head position subsided completely. No nystagmus was perceived and no vertiginous sensation felt by the patient in the provocative head positions.

Though most patients were found to be absolutely symptom free as regards positional vertigo, yet 31 to $64 \%^{7-10} \mathrm{com}$ plained of a new set of symptoms of unsteadiness, dizziness, and either light-headedness or a heavy-headedness and a feeling of persistent postural imbalance when reviewed after 3 to 4 days. Repeated positional tests on all these patients did not show any evidence of BPPV, that is there was no vertigo or nystagmus in any of the positional tests. These new symptoms were then assessed and reviewed with literature available in PubMed and other portals and were categorized as a separate disease entity known as "post-BPPV syndrome" or "residual dizziness after successful CRM" that are recognized medical conditions. On the basis of the published literature after considering the probable etiologies, and the outcome as presented in the different studies, we devised a protocol and managed all patients of BPPV according to this protocol as described below.

Our protocol for management at Vertigo and Deafness Clinic: We collected the demographic data of all the patients such as the age, sex, associated conditions like stress, anxiety, depression, the duration of the symptoms before the treatment, the possible provocating factors, and the affected side. All patients suspected of positional vertigo from the history were first evaluated for the Dizziness Handicap Inventory (DHI) score and the DHI-E score as per response to question nos. $2,5,10,15,18,20,21,22$, and 23 (even before the positional tests were done if the history suggested BPPV). If the DHI-Emotional (DHI-E) score was found to be more than 10 or if there was any evidence of anxiety and/or depression, or if the patient was aged above 65 years (in which case some anxiety is not unexpected), then the patient was first reassured and counseled by us (the author and the assistant neurotologist in the clinic) not the psychologist, once before the positional test and the requisite maneuver were done, but extensively immediately after the maneuver. The doctors explained and educated the patient on the benign nature of the disorder and reassured about the benignness of the condition. All patients are counseled about the possibility of a mild dizziness that may be felt for a few days after the maneuver and explained that the mild dizziness/unsteadiness, if present after the maneuver, is expected to pass off by itself within a week or so. All patients diagnosed with BPPV and treated with the appropriate therapeutic maneuvers for specific canals such as Epley's for the posterior canal, Gufonis's \& Barbeque for the lateral Canal, and Yacovini's maneuver for the anterior canal. For patients having BPPV of the posterior canals, after the Epley's maneuver was done, the patient was instructed to keep the head end raised by approximately 45 degrees whenever the patient wanted to lie supine (e.g., for sleeping at night) and to prevent hyperextension (backward movement) of the head for the next 24 hours. After the maneuvers were done, all patients were called for follow-up after 3 to 4 days and then reevaluation of the symptoms was done; if any persistence of momentary head spinning with change of head position or if there was any presence of nystagmus on the positional tests, then repetition of the CRM was done. Once the patient was completely free from the symptoms of positional vertigo, the following vestibulometric tests were performed namely videonystagmography [VNG] (to assess lateral canals at low frequency of vestibular stimulation, oculomotor functions), video head impulse test (VHIT) (all six semicircular canals at high frequency of vestibular stimulation), vestibular evoked myogenic potential (for assessment of the otolith organs), subjective visual vertical (SVV) (to assess the perception of verticality that is believed to be mainly an utricular function), and posturographic tests, namely stabilometry and craniocorpography. CRMs had to be repeated just two times in 21 patients and thrice in 4 patients out of the total of 178 patients enrolled for the study.

After all positional tests were found negative, the patient was specifically asked about any RD or any balance problems after the CRM and followed up via telephonic calls and patient follow-ups every week for 2 weeks then every 3 weeks for another 6 weeks, that is, four phone calls over a period of 2 months approximately or till the complete resolution of the symptoms which ever was longer. The outcome of these patients was classified into complete recovery (when the patient reported no balance-related symptoms whatsoever) or partial recovery (when the patient complained that there were no vertiginous symptoms on change of head posture but there were some balance-related issues such as dizziness/unsteadiness/light or heavy-headedness). The clinical findings and the history of the patients were then compared with the patient's premaneuver DHI and DHI-E scores as well as the vestibulometric findings detailed in the previous paragraph. For patients with partial recovery, the patient was called over, clinically examined, and the DHI score worked out again. These patients were made to fill up anxiety and depression questionnaires to find out anxiety and depression levels in these patients. In the first visit, these patients were simply reassured and counseled by the author or the junior neurotologist (co-author) and it was explained that this is a self-limiting disease that usually does not require medication and subsides spontaneously within 2 to 3 weeks at the most. If the symptoms persisted for more than 2 weeks, patients were assessed and counseled by the clinical psychologist 
in the clinic, and as per the psychologist's discretion cognitive behavioral therapy was started. In cases where the symptoms persisted more than 2 weeks after professional counseling, a low dose of benzodiazepine and selective serotonin reuptake inhibitor (SSRI) was started and the patient follow-up continued.

\section{Results}

A total of 178 patients diagnosed with geotropic BPPV were included in the study out of which 68 were males and 110

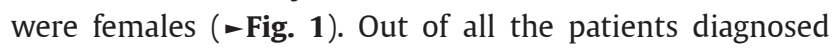
with BPPV, the posterior canal was involved in 150 cases as compared with 37 cases affecting the lateral canals and only in one patient the anterior canal was found to be involved. In five patients, there was multiple canal disorder involving both the posterior and lateral canals. In these five patients, the maneuvers were done in two or three sessions with a gap of 3 days, and the most affected canal was treated first followed by the other canal. Twenty-three patients (13\%) out of 178 ( - Fig. 2) showed RD after successful CRM characterized by unsteadiness, dizziness, and light or heavy-headedness. Unsteadiness as a complaint was mentioned by 14 of the patients suffering from RD after CRM; sinking sensation/ light-headedness was complained by 7 and dizziness were complained by 11 patients out of the 23 who had residual symptoms after successful repositioning maneuvers. Hence, most of these patients of RD after the successful maneuver had multiple complaints. In six (26\%) of the patients, the RD spontaneously subsided within 7 days and in 11 (48\%) of these 23 patients, symptoms were resolved within 2 weeks without any treatment just by reassuring that the symptoms will disappear within the next few days; 6 (26\%) patients complained of the symptoms even after 2 weeks. All these six patients were then sent to the psychologist in the clinic for psychological evaluation and counseling. With just two sessions, three patients were relieved of the symptoms completely, one within 2 weeks (i.e., 4 weeks after the maneuver) and two within 4 weeks (i.e., after 6 weeks of the maneuver and within a month of the start of the psychological sessions). Only in three (13\%) patients, there was chronic persistence of the symptoms where antianxiety drugs and continued psychological counseling were required, leading to complete relief from the symptoms by 3 months. There was no patient where the symptoms of RD persisted above 3 months. No antivertigo drugs, most of which are marketed as vestibular sedatives and some such as betahistine (which are now being touted as vestibular stimulants but was previously known as a vestibular sedative), were used in any of these post-BPPV syndrome patients (-Table $\mathbf{1}$ ).

Correlation with the previous DHI and DHI-E scores showed that 19 of these 23 patients (82.6\%) had a DHI-E score of above 10 which is very much in concurrence with the study of Martellucci et al. All these patients also had a high DHI score above.

\section{Discussion}

There are several possible hypothesis accounting for these new symptoms of nonrotatory dizziness, unsteadiness, and light or heavy-headedness after a successful CRM. Though some researchers have related it to a disorder of the utricle, there is no objective, definitive, and foolproof scientific evidence to establish that post-BPPV syndrome is due to utricular dysfunction. Whether the utricle is pathological, rather malfunctional in post-BPPV syndrome, is a controversial issue. All researches

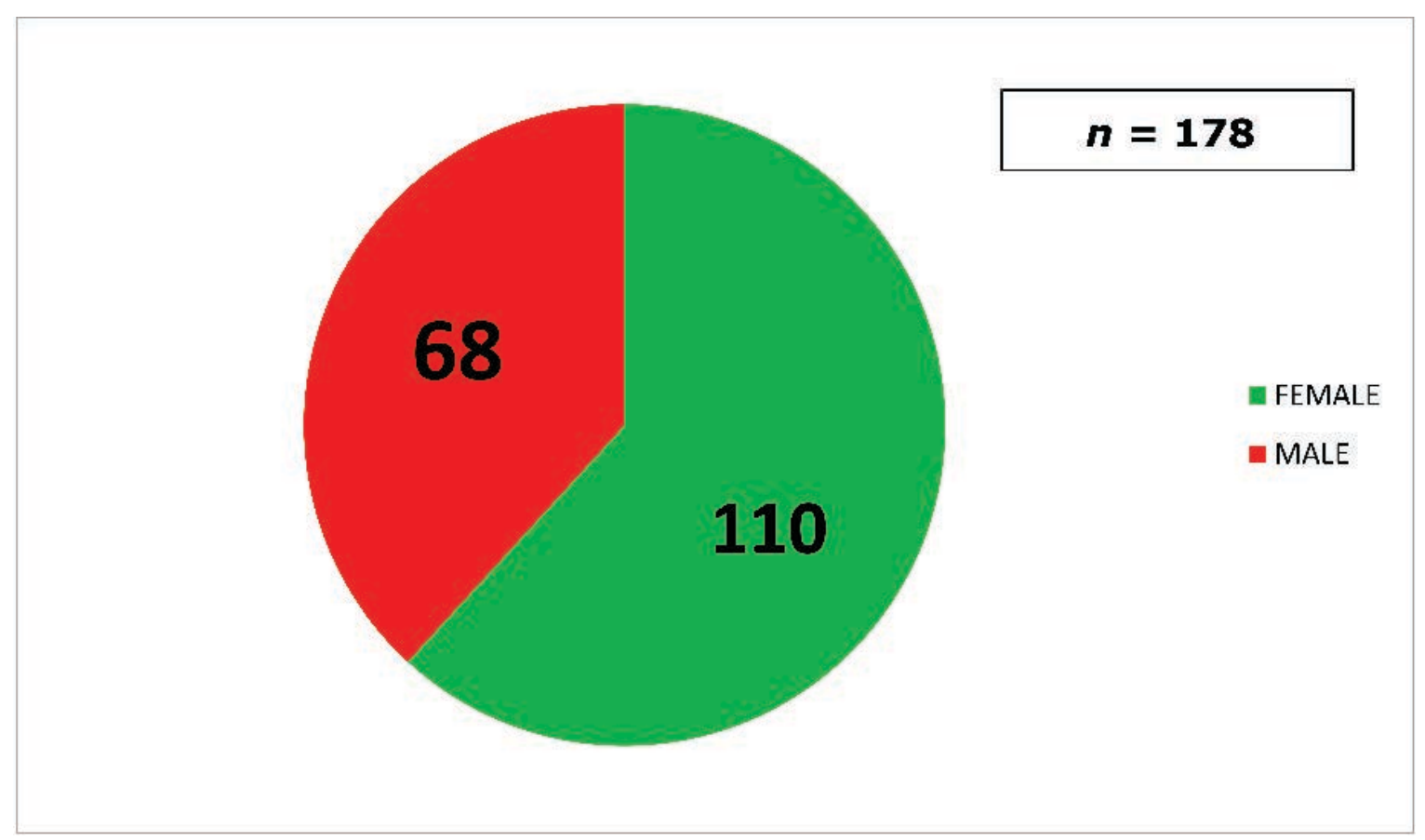

Fig. 1 The sex distribution of the patients of ageotropic benign paroxysmal positional vertigo patients on whom successful canalith repositioning maneuver was done at Vertigo and Deafness Clinic. 


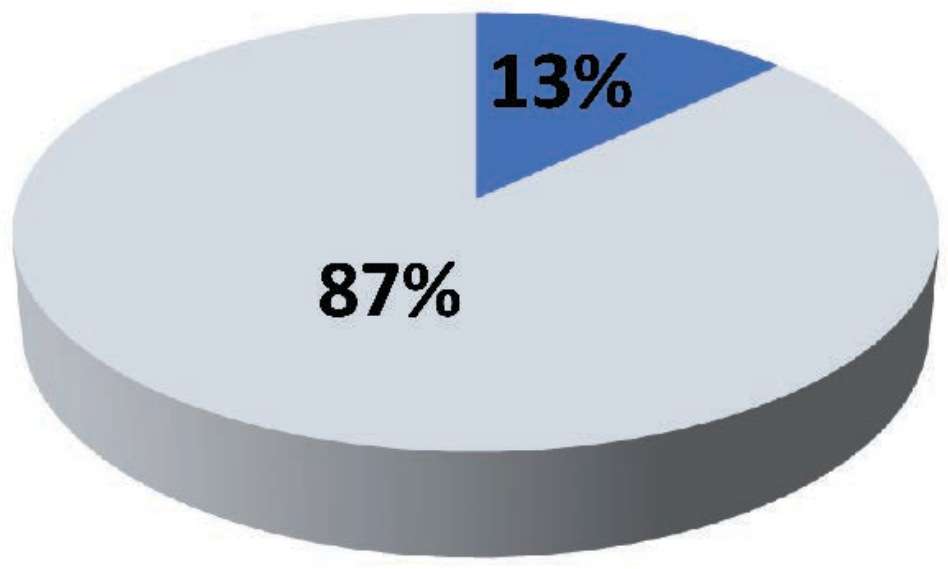

RD after CRM

NO RD after CRM

Fig. 2 The percentage of patients who complained of residual dizziness (RD) after a successful canalith repositioning maneuver (CRM) at Vertigo and Deafness Clinic.

Table 1 Findings of the different vestibulometric tests after the BPPV was corrected by the different liberatory maneuvers

\begin{tabular}{|c|c|c|c|}
\hline \multicolumn{4}{|c|}{ Vestibulometric tests in patients of BPPV after successful CRM } \\
\hline Name of tests & Normal & Abnormal & Percentage abnormal \\
\hline Noncaloric VNG & 177 & 1 & $0.56 \%$ \\
\hline Caloric VNG & 159 & 3 & $1.8 \%$ \\
\hline VHIT & 177 & 1 & $0.56 \%$ \\
\hline Cervical VEMP & $\begin{array}{l}160 \text { (in } 26 \text { patients VEMP } \\
\text { not done due to conduc- } \\
\text { tive hearing loss) }\end{array}$ & 2 & $1.25 \%$ \\
\hline Ocular VEMP & $\begin{array}{l}156 \text { (in } 16 \text { patients VEMP } \\
\text { not done due to conduc- } \\
\text { tive hearing loss) }\end{array}$ & 6 & $3.84 \%$ \\
\hline Stabilometry & 162 & 7 & $4.37 \%$ \\
\hline CCG & 151 & $\begin{array}{l}\text { Wide sway in } 13 \text { deviation / } \\
\text { rotation: } 10\end{array}$ & $15.23 \%$ \\
\hline SVV & 160 & 3 & $1.86 \%$ \\
\hline
\end{tabular}

Abbreviations: BPPV, benign paroxysmal positional vertigo; CCG, craniocarcinography; CRM, canalith repositioning maneuver; SVV, subjective visual vertical; VEMP, vestibular evoked myogenic potential; VHIT, video head impulse test; VNG, videonystagmography.

Note: It is evident that nearly all patients had normal findings in the vestibulometric tests and the incidence of abnormal findings was negligible indicating thereby that vestibular function is essentially normal.

have used the SVV test and the ocular vestibular evoked myogenic potentials (oVEMPs) test to evaluate utricular function in BPPV and in post-BPPV syndrome. But the results of evaluation of utricular function by these conventional tools such as the SVV and the oVEMPs are equivocal, ambiguous and hence scientifically unreliable ${ }^{14}$ as also detailed below. Hence, dependability of these tests to evaluate the utricular function is questionable to say the least. SVV and oVEMP are possibly not very authentic tests of pure utricular function; no wonder different studies on BPPV and post-vestibular syndrome by SVV and oVEMP have shown contradictory results. Whether the contradictory results are due to these being biased studies or the tests themselves are unreliable and fallacious is difficult to say; in all probability it is the latter that is true. Whereas Bohmer and Von Brevern ${ }^{15}$ did not find any abnormality in the SVV test in BPPV and in post-BPPV syndrome, and Farralli ${ }^{16}$ found only sub-clinical abnormality in a few patients and that too only in early onset BPPV, Gall reported SVV abnormalities in $85 \%$ patients of BPPV ${ }^{17}$ and Vanucchhi reported abnormal SVV findings in a clinically significant number of BPPV and post-BPPV syndrome patients. Some of these studies were on BPPV patients prior to and after the maneuvers and some in 
patients with established post-BPPV syndrome. Faralli et al ${ }^{16}$ reported that most post-BPPV syndrome patients had normal utricular function when tested 1 week after the successful maneuver. In fact, Faralli et $\mathrm{al}^{16}$ reported RD was inversely correlated with the deviation of SVV measured a week after efficacious liberatory maneuver, that is, more the RD, less the SVV abnormality. However, both researchers used SVV as the tool to evaluate utricular function that as discussed may not be the best modality to evaluate utricular function. Faralli's work contradicts the hypothesis that otolith dysfunction is the cause of RD after successful CRM in BPPV (post-BPPV syndrome). A relationship between RD and disorder of otolithic organs evaluated by oVEMP was also reported in several studies. ${ }^{15,18,19}$ There is controversy in what is an abnormal oVEMP. Is it the total absence of oVEMP on the ipsilateral side? Is it the delay of the wave peaks at n10 and p15 or just the amplitude difference? As per Mendel, there were no statistically significant changes in the amplitude of p15 latency on the affected side before and after successfully performed repositioning procedures but some increase in amplitude was found after the CRM though not statistically very significant. The unreliability of oVEMP as a test of utricular function is well established in scientific literature. As per the report of the Guideline Development, Dissemination, and Implementation Subcommittee of the American Academy of Neurology on the efficacy of VEMPs published in the December 2017 issue of Neurology-"Evidence is insufficient to determine whether cVEMP and oVEMP can accurately identify vestibular function specifically related to the saccule/utricle." VEMP tests are labeled as "Level U" evidence, that is, inadequate proof of its diagnostic efficacy for otolith organ dysfunction. ${ }^{14}$ Other authors too have opined that as regards the authenticity of SVV as a test of utricular function, scientific evidence is uncertain and ambiguous. ${ }^{19}$ SVV actually assesses perception of verticality that is believed to be determined by combined inputs from semicircular canals, proprioceptors (and possibly the cerebral cortex also), and the otolith organs, not utricle alone. ${ }^{19}$ Hence, SVV too is not an uncontaminated test of utricular function. All these suggest that SVV and oVEMP tests are not dependable tests to evaluate utricular function. Therefore, there is no definitive or authentic and tenable evidence that there is any utricular dysfunction in post-BPPV syndrome as not only are the SVV and oVEMP tests unreliable indices of utricular function, but the results of the different studies too have shown contradictory results. Even if for argument sake the post-BPPV syndrome is accepted to be due to utricular dysfunction and is caused by the stimulation of the utricle by particles sent back from the affected semicircular canals into the utricle as proposed by some researchers such as Prokopakis et $a l,{ }^{21}$ Inukai et $a l,{ }^{22}$ and Inagaki et $a l,{ }^{23}$ yet as per the authors of the hypothesis this may remotely explain the dizziness faced by the patient for the first 2 to 3 days after CRP but cannot account for any symptoms beyond this time as very mild SVV changes but within normal limits have been demonstrated in the first 2 to 3 days after CRM. Moreover, this should occur in all patients in whom the CRM is done as in all patients in whom CRM is successfully done, the dislodged otoconia are sent back to the utricle, and this should not happen only in a selected few patients as post-BPPV syndrome is not reported by all patients in whom CRM is done. In addition, if post-BPPV syndrome is caused by utricular stimulation by the particles repositioned into the utricle by the maneuver, then in severe BPPV with large amount of debris there should be more utricular stimulation after CRM and lead to more RD as more debris are put back into utricle causing more utricular stimulation; however, this does not happen as RD is unrelated to the severity of BPPV. Faralli et $\mathrm{a}^{16}$ concluded that RD after successful maneuvers seems to be unaffected by utricular involvement as found by testing utricular function by SVV. In RD patients, more SVV abnormalities were found in those where the maneuver was done in the first 4 days of the onset of BPPV, in patients who had the BPPV for a longer duration the SVV was normalized indicating that utricular problem, if any, gets resolved quickly and is not a cause of RD which can sometimes persist for weeks. Hence, there is absolutely no scientific basis of the contention that post-BPPV syndrome is caused by some form of utricular overload induced by replacing the dislodged otoconia from the semicircular canals into the utricle. The commonly used term "Utricular Overload" is more of a commercial gimmick used by some pharmaceutical companies to unscrupulously market their antivertigo drugs after BPPV rather than an evidence-based scientific entity.

Some other theories propounded and hypothesized as the pathophysiology of post-BPPV syndrome, although theoretically valid, have not yet been supported by definitive data and are at best conjectural, not adequately evidence based are as follows:

1. Persistence of a small amount of otoconial debris in the affected semicircular canals that are insufficient to cause cupular deflection and hence provoke no nys$\operatorname{tagmus}^{24}$; this is just a theorized explanation without any evidence to substantiate it. Any particles present in the semicircular canals causing some form of stimulation of the semicircular canals would have caused a spinning sensation as stimulation of semicircular canals causes a sensation of spinning that is not there in post-BPPV patients. These patients have dizziness, light or heavy headedness, and postural unsteadiness none of which are actually symptoms of semicircular canal stimulation. Hence, this theory is not tenable.

2. A dysregulation of the sympathoneural system: It is proposed that post-BPPV syndrome is caused by stimulation of the vestibulosympathetic reflex that induces orthostatic hypotension (dysregulation of the vestibulosympathetic system). ${ }^{8,25}$ The contention of this hypothesis is that utricular stimulation by the otoliths inserted from SSC into the utricle by the CRM causes a orthostatic hypotension that is manifested as RD in some patients after CRM. This can at best account for the feeling of light headedness only but not the other symptoms of post-BPPV syndrome. Unsteadiness and dizziness cannot be explained by this hypothesis. Hence, this too is not a very tenable hypothesis on the mechanism for RD after CRM 
3. An incomplete central readaptation as proposed by Faralli et al. ${ }^{16}$ The presence of otoconia in the semicircular canals during BPPV causes erratic stimulation of the vestibular system. This possibly induces some form of adaptation at the level of the vestibular nuclei and in the higher vestibular pathways for the maintenance of proper balance with minimum discomfort. After the reversal of the pathology of BPPV by the CRM, the balance system requires some time in certain patients to readapt and readjust to the old system when there was no BPPV and the delayed readaptation possibly caused the dizziness and unsteadiness that are the main symptoms of post-BPPV syndrome. As this readaptation is a cognitive phenomenon only in some patients not all have RD after the CRM. This again, though completely hypothetical, may be partially acceptable as a cause of the RD after the CRM.

Several studies show correlation between anxiety, fear of fall, and residual symptoms after BPPV. ${ }^{10,26}$ Most published studies and this study too as well as clinical experience have shown that patients with high anxiety and depression as well as aged patients (in all of whom anxiety and depression are more prevalent) are the ones who are much more likely to develop post-BPPV syndrome. In studies where the DHI score of BPPV patients has been recorded prior to the CRM, it has been shown that those patients who have high scores in questions nos. $2,5,10,15,18,20,21,22$, and 23 of the DHI are much more prone to developing post-BPPV syndrome. ${ }^{26}$ These particular questions are all related to emotional issues. Martellucci et al in their study reported that high score in DHI-E in the pre maneuver stage is a strong indicator or predictor of RD. ${ }^{28}$ The items of the DHI-E, in particular, investigate the feelings of insecurity, anxiety, and depression experienced by patients as result of their disorders. Insecurity and anxiety are known to be a strong contributory factor in the genesis of dizziness that is the primary symptom of post-BPPV syndrome. Dizziness in many cases is known to be a somatoform disorder in patients who have experienced events of stress. The intense episodes of paroxysmal vertigo, which characterize BPPV, can themselves represent a very strong stressor event due to the severity and scariness of the symptoms and the unpredictability of vertigo attacks. Several studies correlate anxiety, fear of vertigo recurrence, and residual symptoms after BPPV. All this points to post-BPPV syndrome as the expression of a somatoform disorder. A somatoform disorder is a psychogenic disorder in which a patient experiences physical symptoms that are inconsistent with or cannot be fully explained by any underlying general medical or neurologic condition. Faralli et al ${ }^{29}$ showed that the persistence of RD is correlated with mental stress that in turn is affected by the duration and recurrence of BPPV, age, and gender. Martellucci et $\mathrm{al}^{28}$ reported that BPPV patients who suffer from anxiety disorders show longer lasting and more disabling RD after the resolution of the acute vertigo of BPPV. Anxiety plays an additional contributory role in dizziness that can be considered in some situations as a somatoform disorder caused by stressful events. Mendel et $\mathrm{al}^{30}$ reported that residual feelings of anxiety and depression persisted in patients suffering from peripheral vestibular disorders even after the original peripheral disorder was corrected. As BPPV is a peripheral disease, this applies for BPPV also. There is a loss of self-confidence and a feeling of insecurity that very significantly disturbs daily life.

A very important question here is whether there is actually any documentable postural instability in positional vertigo and in post-BPPV syndrome? Not really. Celebiosy et a $\mathrm{l}^{31}$ had studied posturography in patients of BPPV and post-BP$\mathrm{PV}$ syndrome and found that patients with posterior canal (PC) BPPV had impaired postural balance, that is, abnormal sway before the CRM in conditions where the visual information is unavailable, proprioceptive information is inaccurate, and the postural control relies mostly on vestibular cues. But when visual input was available and/or proprioceptive inputs were available, there was no abnormality in posturography before the CRM in both PC and horizontal canal (HC) BPPV. Even Blatt et al, ${ }^{32}$ Chang et al, ${ }^{33}$ and Di Girolamo et al ${ }^{24}$ have shown similar results during the sensory organization test of posturography. But after a successful CRM, the sway velocity normalized which means that there was no significant sway even when visual input was withdrawn and proprioceptive inputs impaired after the CRM for all the PC-BPPV patients. In horizontal canal BPPV, there is NO abnormal sway before the CRM and even after the CRM, there is no abnormal sway. This means that after the CRM, posturographic studies are normal in both posterior canal BPPV and in lateral canal BPPV. This is a very important finding to understand the pathophysiology of RD after BPPV correction and this has very significant implications on management.

The issue of the so called utricular overload causing any unsteadiness after CRM is hence not a tenable and acceptable cause of post-BPPV syndrome. This needs to be emphasized and settled once and for all that post-BPPV syndrome is not caused by any utricular overload. The issue is reiterated by the fact that in HC BPPV there is no documentable abnormal sway in posturography as shown by the study of Celebisoy et $\mathrm{al}^{31}$ even though in HC BPPV too we are loading the utricle with otoconia from the lateral canal. So, the bottom-line is that (1) issue of utricular dysfunction as a cause of RD is not tenable and (2) there is actually no significant or documentable unsteadiness after a successful CRM even though it is complained of by the patient. Had there been any actual unsteadiness, it would be demonstrable and documentable in the posturographic studies.

Does this lead to the conclusion that RD is a somatoform disorder?

\section{Conclusion}

Well, as this study as well as the different published studies show in all probability, yes. Hence, if the psychic part is taken care of and addressed to from the very beginning even before the requisite maneuvers are done as per the protocol followed in this study, then the incidence of post-BPPV syndrome is much less (just $13 \%$ as compared with the published international figures of 34 to $61 \%$ ). This too indirectly proves that 
post-BPPV syndrome is actually a somatoform disorder and that different hypotheses have been suggested as the pathomechanism of post-BPPV syndrome, such as utricular pathology, utricular overload, dysregulation of the vestibulo-sympathetic system, and delayed re-adaptation, are all merely conjectural hypothesis without any documentable evidence and is bereft of scientific logic as explained in the discussion.

These 23 patients (only 13\%) out of 178 patients of BPPV successfully corrected by the CRMs presented with the complaint of subjective dizziness, that is, nonmotion sensations of disturbed or impaired spatial orientation; lightheadedness, that is, sinking/floating sensation; and unsteadiness which is feeling of being unstable while standing or walking and feelings like a false or distorted sensations of swaying, rocking, bobbing, or bouncing of oneself. None of them complained of spinning of the surroundings (external vertigo) or even a spinning sensation inside the head (internal vertigo) that is present in BPPV patients. These new symptoms could be easily differentiated from the typical head spinning that is a primary feature of a vestibular disorder and had no relation to change of head posture which is the hallmark of BPPV. As already mentioned, repeated positional tests did not show any evidence whatsoever of any positional nystagmus in repeated positional tests. But on detailed questioning, all these patients had one complaint in common that is they have not got back their sense of stability and felt like something about their balance was not right. The morbidity and poor QOL caused by this were in fact more than the actual BPPV as most of these patients said that when they had the positional vertigo, they at least had some control over their disease as they could avoid the vertigo by not bending the head in the provoking position. But now they were persistently in discomfort and had a nagging unsteadiness and dizziness that were no less mentally and physically traumatic than the positional vertigo. There is no denying that post-BPPV syndrome is a medical disorder with high morbidity but it cannot be attributed to any organic deficit/damage to the vestibular labyrinths or for that matter to any one organ in the vestibular system. The normal findings in the detailed vestibular function tests namely VNG, VHIT, SVV, cervical and oVEMP as well as on posturography performed in our clinic on all patients after the BPPV was cured by the maneuvers only do indicate so. None of the patients of post-BPPV syndrome in this study needed any vestibular sedatives and in $74 \%$ the symptoms subsided spontaneously without any medication within 2 weeks just by counseling and by reassurance by the doctors (the two authors who are not qualified in counseling). In the $26 \%$ remaining patients of post-BPPV syndrome half of the patients were completely symptom free just by professional counseling by a qualified psychologist and the rest required low doses of benzodiazepines and SSRIs, no other medication whatsoever. However, in these patients the symptoms matched very much with phobic postural vertigo that is a form of functional dizziness (except that the symptoms subsided within 3 months whereas in phobic postural vertigo the symptoms persist for more than 3 months as per definition of phobic postural vertigo). The study hence reiterates that by following the protocol as done by us in the clinic where counseling and reassurance starts from the time the presenting symptoms suggest BPPV, the outcome is much better, that is, not only is the incidence of post-BPPV syndrome much lesser but the requirement of medication is minimal.

All these prove that (1) the Post BPPV syndrome is primarily a somatoform disorder without any organic damage to the vestibular system, (2) the incidence of this highly morbid disease can be drastically reduced by following the protocol practiced in our clinic which primarily aims at addressing the psychic component only by counseling and reassuring and (3) it is primarily a self-limiting condition and in the very few where treatment is at all needed, low dose of benzodiazepines and SSRI's help by allaying the anxiety and apprehension.

\section{Conflict of Interest}

None declared.

\section{References}

1 von Brevern M, Radtke A, Lezius F, et al. Epidemiology of benign paroxysmal positional vertigo: a population based study. J Neurol Neurosurg Psychiatry 2007;78(7):710-715

2 Baloh RW, Honrubia V, Jacobson K. Benign positional vertigo: clinical and oculographic features in 240 cases. Neurology 1987;37(3):371-378

3 Nedzelski JM, Barber HO, Mcllmoyl L. Diagnoses in a dizziness unit. J Otolaryngol 1986;15(2):101-104

4 Mandalà M, Santoro GP, Awrey J, Nuti D. Vestibular neuritis: recurrence and incidence of secondary benign paroxysmal positional vertigo. Acta Otolaryngol 2010;130(5):565-567

5 Hemenway WG, Lindsay JR. Postural vertigo due to unilateral sudden partial loss of vestibular function. Ann Otol Rhinol Laryngol 1956;65(3):692-706

6 Jang YS, Hwang CH, Shin JY, Bae WY, Kim LS. Age-related changes on the morphology of the otoconia. Laryngoscope 2006;116(6):996-1001

7 Seok JI, Lee HM, Yoo JH, Lee DK. Residual dizziness after successful repositioning treatment in patients with benign paroxysmal positional vertigo. J Clin Neurol 2008;4(3):107-110

$8 \mathrm{Kim} \mathrm{HA}$, Lee H. Autonomic dysfunction as a possible cause of residual dizziness after successful treatment in benign paroxysmal positional vertigo. Clin Neurophysiol 2014;125(3):608-614

9 Jung HJ, Koo JW, Kim CS, Kim JS, Song JJ. Anxiolytics reduce residual dizziness after successful canalith repositioning maneuvers in benign paroxysmal positional vertigo. Acta Otolaryngol 2012;132(3):277-284

10 Teggi R, Quaglieri S, Gatti O, Benazzo M, Bussi M. Residual dizziness after successful repositioning maneuvers for idiopathic benign paroxysmal positional vertigo. ORL J Otorhinolaryngol Relat Spec 2013;75(2):74-81

11 Baloh RW, Jacobson K, Honrubia V. Horizontal semicircular canal variant of benign positional vertigo. Neurology 1993;43(12):2542-2549

12 Ganança FF, Gazzola JM, Ganança CF, Caovilla HH, Ganança MM, Cruz OL. Elderly falls associated with benign paroxysmal positional vertigo. Rev Bras Otorrinolaringol (Engl Ed) 2010;76(1):113-120

13 Chu CH, Liu CJ, Lin LY, Chen TJ, Wang SJ. Migraine is associated with an increased risk for benign paroxysmal positional vertigo: a nationwide population-based study. J Headache Pain 2015;16:62

14 Fife TD, Colebatch JG, Kerber KA, et al. Practice guideline: Cervical and ocular vestibular evoked myogenic potential testing. Report of the Guideline Development, Dissemination, and 
Implementation Subcommittee of the American Academy of Neurology. Neurology 2017; 89(22):2288-2296

15 von Brevern M, Schmidt T, Schönfeld U, Lempert T, Clarke AH. Utricular dysfunction in patients with benign paroxysmal positional vertigo. Otol Neurotol 2006;27(1):92-96 2017

16 Faralli M, Lapenna R, Giommetti G, Pellegrino C, Ricci G. Residual dizziness after the first BPPV episode: role of otolithic function and of a delayed diagnosis. Eur Arch Otorhinolaryngol 2016;273(10):3157-3165

17 Gall RM, Ireland DJ, Robertson DD. Subjective visual vertical in patients with benign paroxysmal positional vertigo. J Otolaryngol 1999;28(3):162-165

18 Yetiser S, Ince D, Gul M. An analysis of vestibular evoked myogenic potentials in patients with benign paroxysmal positional vertigo. Ann Otol Rhinol Laryngol 2014;123(10):686-695

19 Seok, Shiraishi K, Kobayashi T, et al. benResidual dizziness after successful treatment of idiopathic ign paroxysmal positional vertigo originates from persistent utricular dysfunction. Acta Oto-Laryngologica 2017;137(11): 1149-1152

20 Giorgia Giommetti, Ruggero Lapenna, Roberto Panichi, et al. Residual dizziness after successful repositioning maneuver for idiopathic benign paroxysmal positional vertigo: a review. Audiology Research 2017;7:178

21 Prokopakis EP, Lachanas VA, Christodoulou PN, Velegrakis GA. Dizziness after canalith repositioning procedure for benign paroxysmal positional vertigo. Auris Nasus Larynx 2007;34(3):435, authorreply 437

22 Inukai K, Koizuka I, Takahashi S. Head-tilting stabilometry in patients with benign paroxysmal positional vertigo. Ann Otol Rhinol Laryngol 2014;123:686-695

23 Inagaki T, Suzuki M, Otsuka K, et al. Model experiments of BPPV using isolated utricle and posterior semicircular canal. Auris Nasus Larynx 2006;33(2):129-134

24 Di Girolamo S, Ottaviani F, Scarano E, Picciotti P, Di Nardo W. Postural control in horizontal benign paroxysmal positional vertigo. Eur Arch Otorhinolaryngol 2000;257(7):372-375
25 Yates BJ, Miller AD. Properties of sympathetic reflexes elicited by natural vestibular stimulation: implications for cardiovascular control. J Neurophysiol 1994;71(6):2087-2092

26 Teggi R, Giordano L, Bondi S, Fabiano B, Bussi M. Residual dizziness after successful repositioning maneuvers for idiopathic benign paroxysmal positional vertigo in the elderly. Eur Arch Otorhinolaryngol 2011;268(4):507-511

27 Magliulo G, Bertin S, Ruggieri M, Gagliardi M. Benign paroxysmal positional vertigo and post-treatment quality of life. Eur Arch Otorhinolaryngol 2005;262(8):627-630

28 Martellucci S, Pagliuca G, de Vincentiis M, et al. Features of residual dizziness after canalith repositioning procedures for benign paroxysmal positional vertigo. Otolaryngol Head Neck Surg 2016;154(4):693-701

29 Faralli M, Ricci G, Ibba MC, Crognoletti M, Longari F, Frenguelli A. Dizziness in patients with recent episodes of benign paroxysmal positional vertigo: real otolithic dysfunction or mental stress? J Otolaryngol Head Neck Surg. 2009;38(3):375-380

30 Mendel B, Bergenius J, Langius A. Dizziness symptom severity and impact on daily living as perceived by patients suffering from peripheral vestibular disorder. Clin Otolaryngol Allied Sci 1999;24(4):286-293

31 Celebisoy N, Bayam E, Güleç F, Köse T, Akyürekli O. Balance in posterior and horizontal canal type benign paroxysmal positional vertigo before and after canalith repositioning maneuvers. Gait Posture 2009;29(3):520-523

32 Blatt PJ, Georgakakis GA, Herdman SJ, Clendaniel RA, Tusa RJ. The effect of the canalith repositioning maneuver on resolving postural instability in patients with benign paroxysmal positional vertigo. Am J Otol 2000;21(3):356-363

33 Chang WC, Hsu LC, Yang YR, Wang RY. Balance ability in patients with benign paroxysmal positional vertigo. Otolaryngol Head Neck Surg 2006;135(4):534-540 